\title{
Anticancer effect of salidroside reduces viability through autophagy/PI3K/Akt and MMP-9 signaling pathways in human bladder cancer cells
}

\author{
TIAN LI ${ }^{1,2}$, KEWEI XU ${ }^{3}$ and YIFAN LIU ${ }^{1,2}$ \\ ${ }^{1}$ Department of Urology, The Fifth Affiliated Hospital of Guangzhou Medical University; \\ ${ }^{2}$ Minimally Invasive Technique and Product Translational Center, Guangzhou Medical University, \\ Guangzhou, Guangdong 510700; ${ }^{3}$ Department of Urology, The Second Affiliated Hospital of Sun Yat-Sen University, \\ Guangzhou, Guangdong 510120, P.R. China
}

Received November 11, 2016; Accepted January 23, 2018

DOI: $10.3892 / \mathrm{ol} .2018 .8982$

\begin{abstract}
Salidroside has a wide range of pharmacological activities, including antitumor, anti-inflammatory, analgesic, antibacterial, antiviral and anti-fertility abilities. In the present study, the effects of salidroside on the viability and apoptosis of bladder cancer cells, and the potential underlying mechanisms, were examined. In the present study, treatment with salidroside reduced cell viability, and induced apoptosis and caspase-9/3 activation in the T24 human bladder carcinoma cell line. Salidroside induced autophagy, promoted the protein expression of nucleoporin p62 and the microtubule-associated proteins $1 \mathrm{~A} / 1 \mathrm{~B}$ light chain $3 \mathrm{~B}$, suppressed phosphoinositide 3-kinase (PI3K) and phosphorylated protein kinase $\mathrm{B}$ ( $\mathrm{p}-\mathrm{Akt})$ expression, inhibited matrix metalloproteinase-9 (MMP-9) expression and increased that of Bcl-2-associated X protein, which functions as an apoptosis regulator in T24 cells. In the present study, it was demonstrated that the effect of salidroside reduced the viability and induced the apoptosis of bladder cancer cells through the autophagy/PI3K/Akt and MMP-9 signaling pathways.
\end{abstract}

\section{Introduction}

In 2008, the incidence of bladder cancer was 7.49/100,000 according to the National Cancer Registry, and the standardized global incidence was 4.53/100,000 (1). The incidence of bladder cancer was ranked first among all genitourinary cancer types in the Chinese male population and eighth of all malignant tumor types in China, and accounted for $2.50 \%$ of

Correspondence to: Dr Tian Li, Department of Urology, The Fifth Affiliated Hospital of Guangzhou Medical University, 621 Gangwan Road, Guangzhou, Guangdong 510700, P.R. China

E-mail: tianliedit@163.com

Key words: salidroside, bladder cancer, autophagy, phosphoinositide 3-kinase, matrix metalloproteinase-9 all malignant tumors diagnosed in China in 2008 (2,3). For Chinese individuals between 0 and 74 years old, the cumulative incidence of bladder cancer was $0.52 \%$ in 2008 (4).

Autophagy refers to the process in which damaged, degenerated or aging proteins or organelles in cells are transported to lysosomes for digestion and degradation (5). The occurrence of autophagy requires the multiple stages. First, under the regulation of initial autophagic signals, the precursors of autophagy with a cup-shaped double layer coating are formed in the cytoplasm (6). Then, the precursors of autophagy are gradually lengthened, assisted by autophagic proteins. The lengthened precursors of autophagy cover degradative substrates and finally form completely closed autophagic vacuoles (7). Finally, the autophagic vacuoles reach the lysosomes through the intracellular transport system. The adventitia of the autophagic vacuoles integrates with lysosomal membranes and degrades their wrapping via the lysosomes hydrolase activity (7).

Under normal physiological conditions, autophagy is beneficial in maintaining a self-stabilized state (8). When there is stress, autophagy may prevent the accumulation of toxic or carcinogenic proteins and organelles and inhibit cellular cancerization (9). However, a number of previous studies have indicated that, following neoplasia, autophagy may provide more abundant nutrition for cancer cells and promote tumor growth $(8,9)$. Therefore, in the process of tumorigenesis and tumor development, autophagy serves an important function.

The suppressed phosphoinositide 3-kinase (PI3K)/phosphorylated protein kinase B (p-Akt) signaling pathway exerts anti-apoptosis activity by influencing multiple downstream effector molecules (10). At present, by knocking out or inhibiting P13K/Akt and other relevant genes using gene intervention techniques or micromolecule drugs, the activation of multiple downstream anti-apoptotic effector molecules may be suppressed, in order to promote apoptosis (11). This topic has already become a central focus of cancer research.

Matrix metalloproteinases (MMPs) are a group of zinc ion-containing endopeptidases, able to degrade the extracellular matrix, and to promote tumor invasion and metastasis; the most notable MMPs include MMP-2, MMP-3 and 
MMP-9 (12-14). MMP-2 and MMP-9 are two subtypes of type IV collagenase highly expressed in various tumor types, including breast cancer, ovarian cancer and nasopharyngeal carcinoma, and associated with the degree of invasion and malignancy (12-14). Quantitative detection revealed that MMP-2 and MMP-9 are significantly high in bladder cancer tissues, as compared with in the corresponding control tissues; and invasive tumor types have higher expression levels of these proteins compared with superficial tumor types $(15,16)$.

Of the genes involved in the regulation of apoptosis, the B-cell lymphoma 2 (Bcl-2) family is the most important when it comes to regulating apoptosis. Bcl-2 and Bcl-2-associated X protein (Bax) are, respectively, two of the main anti-apoptotic and pro-apoptotic regulators, which mediate apoptosis via influencing mitochondrial function (17). The BCL2 gene was originally cloned from the local breaking point of chromosome 18 of follicular B-cell lymphoma $(\mathrm{Bcl})$, which is an apoptosis-suppressing gene (18). The $\mathrm{Bcl}-2$ protein is located in the mitochondria, at the nuclear membrane and in the endoplasmic reticulum (18). As increased intracellular calcium concentration is an early indicator of apoptosis, Bcl-2 inhibits apoptosis by preventing this elevation in plasma calcium. In previous years, it was revealed that $\mathrm{Bcl}-2$ expression is the most notable feature in human malignant tumor types, is widely recognized as an anti-apoptotic factor, and is involved in the tumorigenesis through the inhibition of apoptosis and by prolonging cell survival (19). In the extrinsic apoptosis pathway, caspase- 9 and -3 are key signaling transduction pathway proteins (20). Caspase- 9 and -3 form the death-inducing signaling complex for human bladder cancer via the activation of caspase- 8 in apoptotic cell death (20).

Salidroside has anti-inflammatory, antibacterial, antiviral, anti-fertility effects amongst other activities; it is currently used for the treatment of rheumatoid arthritis, blood diseases and skin diseases, and is additionally used as an agricultural insecticide $(21,22)$. However, the anticancer effect of salidroside in suppressing the viability of human bladder cancer cells has not yet been fully clarified. In the present study, the effects of salidroside on the viability and apoptosis of bladder cancer cells, and its potential underlying mechanisms, were examined.

\section{Materials and methods}

Chemicals and reagents. RPMI-1640 medium was purchased from Gibco (Thermo Fisher Scientific, Inc., Waltham, MA, USA). Fetal bovine serum (FBS) and MTT bromide were purchased from Invitrogen (Thermo Fisher Scientific, Inc.). Caspase-9 Activity Assay kit and Caspase-3 Activity Assay kit were purchased from Nanjing KeyGen Biotech Co., Ltd (Nanjing, China) and an Enhanced BCA Protein Assay kit was purchased from Beyotime Institute of Biotechnology (Haimen, China). Salidroside was purchased from Sigma-Aldrich (Merck KGaA, Darmstadt, Germany).

Cell culture. Human bladder cancer T24 cells were obtained from Guangzhou Medical University (Guangzhou, Guangdong) and were maintained in RPMI-1640 medium supplemented with $10 \% \mathrm{FBS}$ in a humidified atmosphere of $5 \% \mathrm{CO}_{2}$ at $37^{\circ} \mathrm{C}$.
MTT assays. T24 cells were seeded into 96-well dishes at a density of $1 \times 10^{4}$ cells/well, and treated with $0,0.5,1$ and $2 \mu \mathrm{M}$ salidroside for 24,48 and $72 \mathrm{~h}$ each. Cell viability was determined using an MTT assay. T24 cells were supplemented with $10 \mu \mathrm{MTT}$ and incubated at $37^{\circ} \mathrm{C}$ for $4 \mathrm{~h}$ in a humidified atmosphere containing 5\% $\mathrm{CO}_{2}$. Once the RPMI-1640 was removed, $150 \mu \mathrm{l}$ dimethyl sulfoxide (DMSO) was added to solubilize the formazan crystals. The optical density of each well was read at $490 \mathrm{~nm}$.

Cell apoptosis assay. T24 cells were seeded into 6-well plates at a density of $1 \times 10^{6}$ cells/well, and then treated with $0.5,1$ and $2 \mu \mathrm{M}$ salidroside for $48 \mathrm{~h}$. The apoptosis of T24 cells was evaluated using a FACScan flow cytometer (BD Biosciences, San Jose, CA, USA). Cells were washed with PBS for 5 min three times and resuspended in $500 \mu \mathrm{l}$ Annexin V Binding Buffer (BD Biosciences). Then, cells were stained with FITC-Annexin V and propidium iodide (BD Biosciences) in the dark for $15 \mathrm{~min}$ at room temperature. Apoptotic cells were analyzed using ImageLab 3.0 (Bio-Rad Laboratories, Inc., Hercules, CA, USA).

Caspase-9/3 activation. T24 cells were seeded into 6-well plates $\left(1 \times 10^{6}\right.$ cells/well), and then treated with $0.5,1$ and $2 \mu \mathrm{M}$ salidroside for $48 \mathrm{~h}$. The activation of caspase-9/3 in T24 cells was evaluated using ELISA colorimetric kits (cat nos. C1157 and C1115; Nanjing KeyGen Biotech Co., Ltd.) according to the manufacturer's protocol. The Caspase-9 inhibitor Z-LEHDFMK (2 mM; Nanjing KeyGen Biotech Co., Ltd.) and the Caspase-3 inhibitor Z-DEVD-FMK (2 mM; Nanjing KeyGen Biotech Co., Ltd.) were added into each well. The optical density of each well was read at $450 \mathrm{~nm}$.

Western blot analysis. T24 cells were seeded in 6-well dishes at $1 \times 10^{6}$ cells/well, and treated with $0.5,1$ and $2 \mu \mathrm{M}$ salidroside for $48 \mathrm{~h}$. T2 4 cells were incubated with ice-cold radioimmunoprecipitation assay buffer (Nanjing KeyGen Biotech Co., Ltd.) for $30-50 \mathrm{~min}$ on ice at $4^{\circ} \mathrm{C}$. Protein concentration was determined using an Enhanced BCA Protein Assay kit (Nanjing KeyGen Biotech Co., Ltd.). Protein extracts $(50 \mu \mathrm{g}$ per lane) were separated via 10\% SDS-PAGE and then transferred to polyvinylidene fluoride membranes (Bio-Rad Laboratories, Inc., Hercules). The blotted membranes were blocked with Tris-buffered saline (TBS) containing $5 \%$ non-fat milk to block nonspecific binding sites for $1 \mathrm{~h}$ at $37^{\circ} \mathrm{C}$. Then, the membranes were incubated with anti-p62 (cat. no. sc-25523; 1:100), anti-PI3K (cat. no. sc-7174; 1:1,000), anti-MMP-9 (cat. no. sc-10737; 1:1,000), and anti-Bax (cat. no. sc-6236; 1:2,000; all Santa Cruz Biotechnology, Inc., Dallas, TX, USA), anti-LC3 (cat. no. 3868; 1:1,000), anti-Akt (cat. no. 4685; 1:2,000) and anti-p-Akt (cat. no. 4060; 1:1,000; both Cell Signaling Technology, Inc., Danvers, MA, USA), and anti-GAPDH (cat. no. sc-25778, 1:500; Santa Cruz Biotechnology, Inc.) overnight at $4^{\circ} \mathrm{C}$. Subsequent to washing in TBS with $0.1 \%$ Tween-20 for 5 min 3 times, the membranes were incubated with a horseradish peroxidase-conjugated sheep anti-mouse immunoglobulin G (cat. no. sc-2004; 1:5,000; Santa Cruz Biotechnology, Inc.) for $1 \mathrm{~h}$ at room temperature. The proteins were detected using enhanced chemiluminescence (Tiangen Biotech Co., Ltd., Beijing, China), and quantified with Image Lab v.3.0 (Bio-Rad Laboratories, Inc.). 


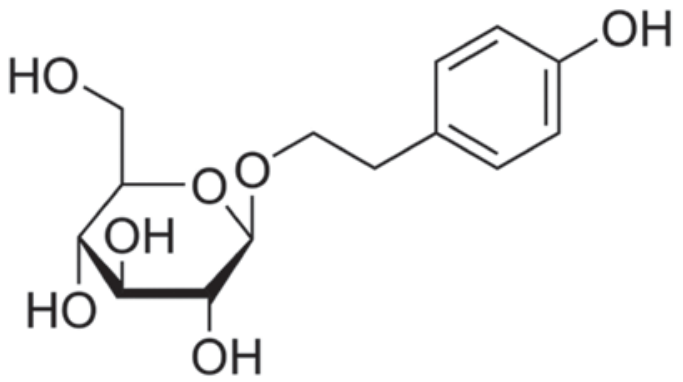

Figure 1. Chemical structure of salidroside.

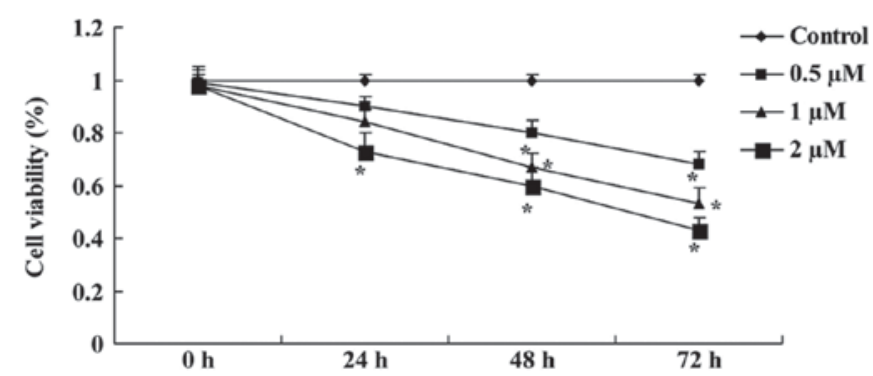

Figure 2. Salidroside suppresses the viability of bladder cancer cells. Viability of T24 cells when treated with salidroside $(0.5,1$ and $2 \mu \mathrm{M})$ was analyzed using MTT assays. "P $<0.05$ vs. the control group $(0 \mu \mathrm{M}$ salidroside $)$.

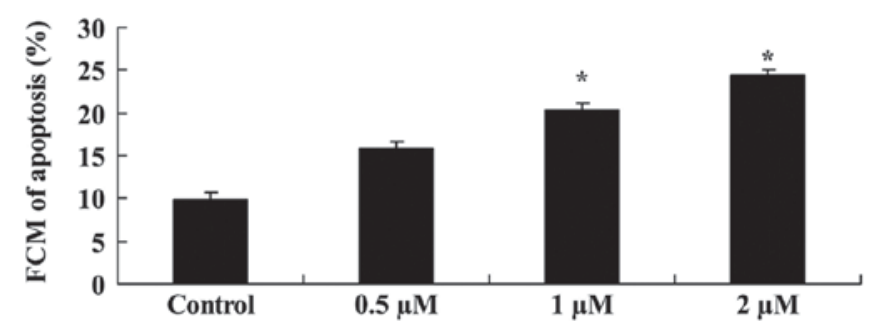

Figure 3. Salidroside induces bladder cancer cell apoptosis. Apoptosis of T24 cells treated with various concentrations of salidroside $(0.5,1$ and $2 \mu \mathrm{M})$ was investigated. " $\mathrm{P}<0.05$ vs. the control group. FCM, flow cytometry.

Statistical analysis. Statistical analysis was performed using SPSS v.18.0 software (SPSS, Inc., Chicago, IL, USA), and performed using an unpaired one-way analysis of variance followed by Tukey's post hoc test. Data are expressed as the mean \pm standard deviation. $\mathrm{P}<0.05$ was considered to indicate a statistically significant difference.

\section{Results}

Salidroside suppresses the viability of bladder cancer cells. The chemical structure of salidroside (purity $>98 \%$ ) is illustrated in Fig. 1. To investigate whether salidroside could suppress the viability of T24 cells, the cells were treated with various concentrations of salidroside $(0.5,1$ and $2 \mu \mathrm{M})$ and then evaluated using an MTT assay. Treatment with salidroside $(0.5,1$ and $2 \mu \mathrm{M})$ significantly suppressed the viability of T2 4 cells at 48 and $72 \mathrm{~h}$ compared with the control $(\mathrm{P}<0.05$; Fig. 2A). Additionally, the administration of $2 \mu \mathrm{M}$ salidroside for $24 \mathrm{~h}$ significantly suppressed the viability of T24 cells compared with the control $(\mathrm{P}<0.05$; Fig. $2 \mathrm{~A})$. The results of the
MTT assay revealed that salidroside also inhibited T24 cell viability (Fig. 2B).

Salidroside induces bladder cancer cell apoptosis. The anticancer effect of salidroside on the cell apoptosis of T24 cells was investigated. T24 cells were treated with different concentrations of salidroside $(0.5,1$ and $2 \mu \mathrm{M})$ and then evaluated using a flow cytometer. As presented in Fig. 3, 1 and $2 \mu \mathrm{M}$ of salidroside significantly induced the apoptosis of T24 cells in a dose-dependent manner, compared with the control group $(\mathrm{P}<0.05)$.

Salidroside induces caspase-9/3 activation in bladder cancer cells. As indicated in Fig. 4, treatment with $0.5 \mu \mathrm{M}$ salidroside for $48 \mathrm{~h}$ non-significantly increased caspase-9/3 activation in T24 cells, compared with in the control group. However, the administration of 1 and $2 \mu \mathrm{M}$ salidroside for $48 \mathrm{~h}$ significantly induced caspase-9/3 activation in T24 cells, compared with in the control group $(\mathrm{P}<0.05)$.

Salidroside induces the autophagy of bladder cancer cells. The anticancer effect of salidroside on the autophagy of T24 cells was investigated. T24 cells were stained with anti-LC3. As presented in Fig. 5, treatment with $0.5,1$ and $2 \mu \mathrm{M}$ salidroside for $48 \mathrm{~h}$ increased the autophagy of T24 cells in a dose-dependent manner, compared with the control.

Salidroside induces $p 62$ and LC 3 protein expression in bladder cancer cells. Next, the effect of salidroside on the autophagy of T24 cells was further investigated by examining the protein expression of p62 and LC3, which are known biomarkers of autophagy. The results indicated that 1 and $2 \mu \mathrm{M}$ of salidroside significantly induced p62 and LC3 protein expression in T24 cells in a dose-dependent manner, compared with in the control $(\mathrm{P}<0.05$; Fig. 6).

Salidroside suppresses PI3K and Akt protein expression in bladder cancer cells. PI3K and Akt protein expression in bladder cancer cells was assessed in order to elucidate the mechanism by which salidroside induces autophagy in T24 cells. PI3K and p-Akt protein expression levels were significantly suppressed by 1 and $2 \mu \mathrm{M}$ salidroside in T24 cells in a dose-dependent manner, compared with in the control ( $\mathrm{P}<0.05$; Fig. 7).

Salidroside suppresses the MMP-9 expression levels of bladder cancer cells. As depicted in Fig. 8A and B, treatment with $0.5 \mu \mathrm{M}$ salidroside for $48 \mathrm{~h}$ non-significantly suppressed MMP-9 protein expression in T24 cells, compared with in the control group. Additionally, the administration of 1 and $2 \mu \mathrm{M}$ salidroside for $48 \mathrm{~h}$ significantly suppressed the MMP-9 protein expression levels in T24 cells, compared with in the control group $(\mathrm{P}<0.05)$.

Salidroside induced Bax expression in bladder cancer cells. As indicated in Fig. 8, treatment with $0.5 \mu \mathrm{M}$ salidroside for $48 \mathrm{~h}$ non-significantly induced Bax protein expression in T24 cells, compared with the control group. However, the administration of 1 and $2 \mu \mathrm{M}$ salidroside for $48 \mathrm{~h}$ significantly increased Bax protein expression in T2 4 cells, compared with in the control group $(\mathrm{P}<0.05)$. 

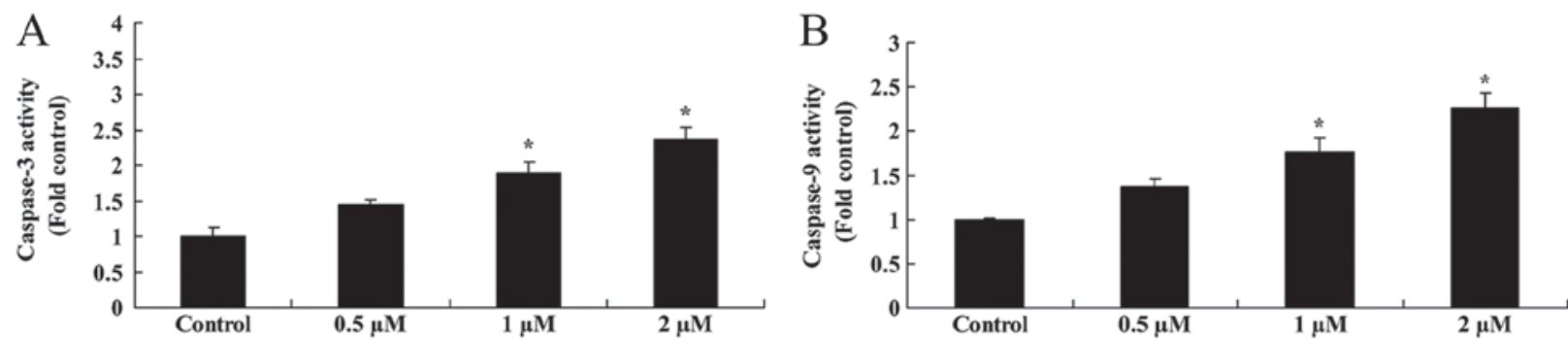

Figure 4. Salidroside induces caspase-9/3 activation in bladder cancer cells. Salidroside was revealed to induce (A) caspase-3 and (B) caspase-9 activation in T24 cells. ${ }^{*} \mathrm{P}<0.05$ vs. the control group.
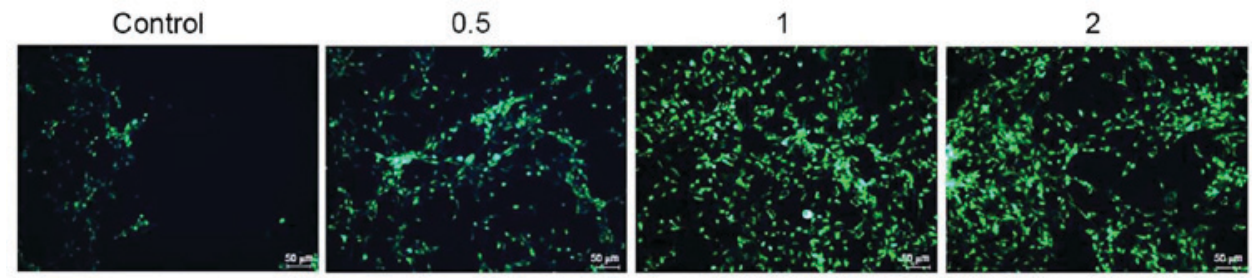

Figure 5. Salidroside induces the autophagy of bladder cancer cells. Various concentrations of salidroside $(0.5,1$ and $2 \mu \mathrm{M})$ were revealed to increase the autophagy of T24 cells in a dose-dependent manner, compared with in the control group.

\section{Discussion}

Bladder cancer is a common malignant tumor of the urinary system. According to World Health Organization statistics on global cancer epidemic diseases, there were 386,300 new cases globally in 2008 , with a 14 -fold variance in the incidence of bladder cancer in certain regions (23). The regions with the highest bladder cancer incidence were Europe and North America (with a male incidence rate of $\geq 20 / 100,000$ and a female incidence rate of up to $5 / 100,000)(24)$. The region with the lowest incidence was Central Africa (incidence rate for males of 1.5/100,000 and an incidence rate for females of $0.3 / 100,000)(25)$. Previous studies revealed that salidroside significantly suppressed the invasion of and induced the apoptosis of human breast cancer (25) and head and neck cancer cells (26). In the present study, the administration of salidroside significantly suppressed the viability of and significantly increased caspase-9/3 activation in human bladder cancer T24 cells, compared with in the untreated cells $(\mathrm{P}<0.05)$.

Autophagy is a normal and ubiquitous physiological process that represents an active defensive strategy for external pessimal stimulation (8); it is also an important form of programmed cell death. Autophagy additionally participates in the pathological development of multiple diseases, including malignant tumor types (27). LC3 is a notable gene that participates in the formation of the autophagosome (9). In the degradation process of autophagic lysosomes, it serves the role of a tumor suppressor; LC3 is an autophagic landmark gene and one of the diagnostic indices of autophagic specificity (9). In the present study, it was revealed that salidroside significantly increased the autophagy of T24 cells in a dose-dependent manner $(\mathrm{P}<0.05)$. Fan et al $(21)$ suggested that salidroside induced apoptosis and autophagy in human colorectal cancer cells through inhibition of the PI3K/Akt/mechanistic target of rapamycin (mTOR) pathway.
LC3 is the gene isogeny of yeast and autophagy-related protein 8 (Atg8) in mammalian cells (28). It has $\sim 30 \%$ amino acid isogeny with Atg8 (28). LC3 is primarily located in the front autophagic vacuoles and the surface of autophagic vacuoles in the cell interior (28). LC3 that participates in autophagic formation may be divided into type-I and type-II (28). Prior to the presentation of autophagy, LC3 generated in cells is modified and processed to become the regularly expressed, soluble type-I LC3 in the cytoplasm (28). When autophagy occurs, type-I LC 3 is modified and processed via ubiquitin-type processing (29). Type-I LC3 forms type-II LC3 by combining with phosphatidyl ethanolamine on the surface of an autophagic membrane; type-II LC3 is always combined on the membrane of intracellular autophagic vacuoles (29). Evaluating the difference in LC3 expression between tumor and normal tissues may provide insight into tumorigenesis and development (9). In the present study, salidroside significantly induced p62 and LC3 protein expression in T24 cells, in a dose-dependent manner, compared with in the control cells $(\mathrm{P}<0.05)$.

Under the stimulation of hunger, hormones, chemicals and ultraviolet light, a double-layer coating is independently formed around substances to be degraded $(30,31)$. The key regulatory factor in this stage is TOR, a serine-threonine kinase known as mTOR in mammals (30). It serves as a receptor for various amino acids, ATP and hormones in cells and may lower their levels (31). It also may inhibit the occurrence of autophagy. When there is nutritional sufficiency and the presence of growth factors, it serves as a primary anti-autophagy signal (28). TOR kinase may phosphorylate the associated gene Atg13 (28). The affinity of phosphorylated Atg13 and Atg1 is reduced and blocks the formation of the Atg13-Atg1 complex, reducing the occurrence of autophagy (31). PI3K/Akt signaling may inhibit the activity of mTOR kinase, thus promoting the activity of growth factors and inhibiting autophagy (32). Conversely, type-III PI3K may promote intracellular substances to be degraded by wrapping by the double layer coating structure 


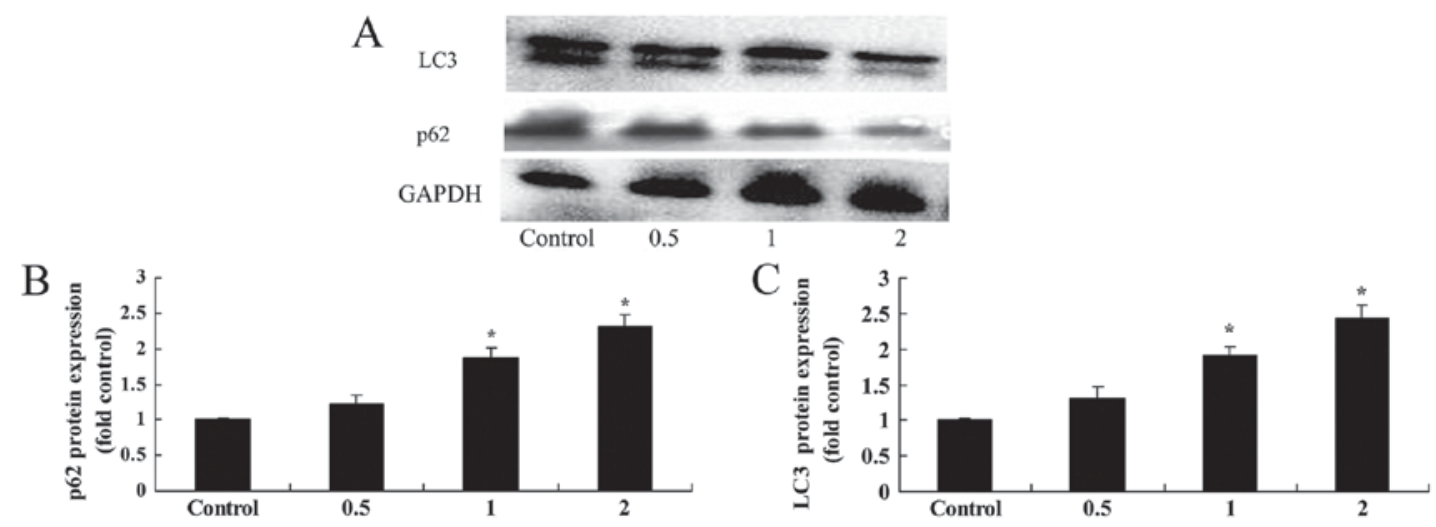

Figure 6. Salidroside induces p62 and LC3 protein expression in bladder cancer cells. (A) Salidroside was revealed to induce p62 and LC3 expression in T24 cells, as determined via western blotting. The protein expression levels of (B) p26 and (C) LC3 were quantified. "P<0.05, compared with the control group. p62, nucleoporin p62; LC3, microtubule-associated proteins 1A/1B light chain 3B.

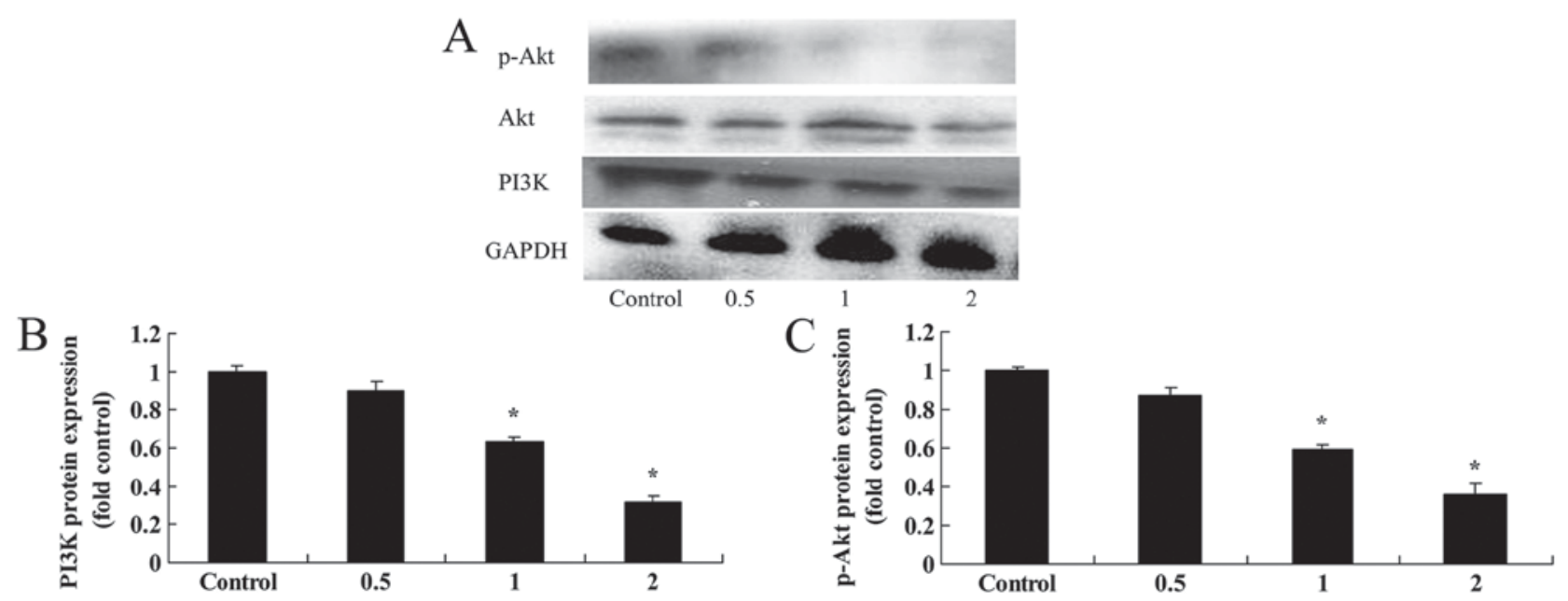

Figure 7. Salidroside suppresses PI3K and Akt protein expression in bladder cancer cells. (A) Salidroside suppresses PI3K and Akt protein expression, as revealed via western blotting. The protein expression levels of (B) PI3K and (C) Akt were quantified. "P $<0.05$, vs. the control group. PI3K, phosphoinositide 3-kinase; Akt, protein kinase B; p-, phosphorylated.

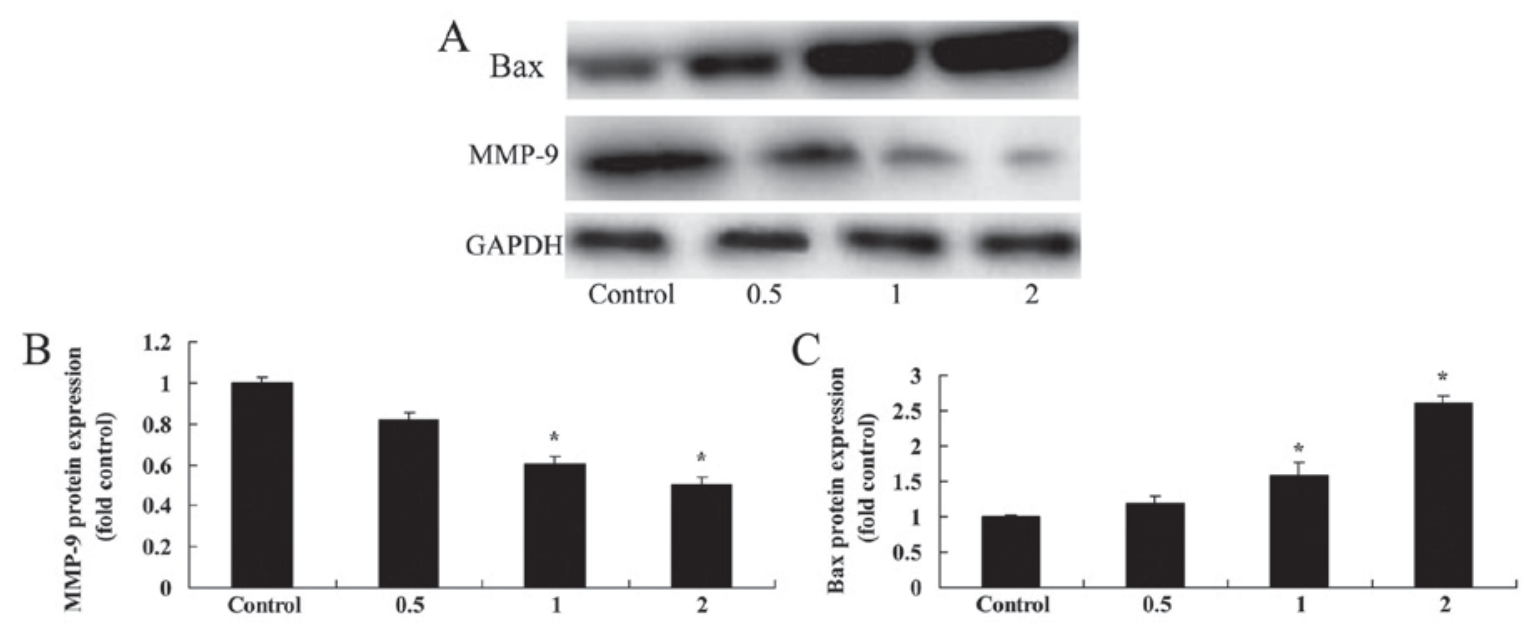

Figure 8. Salidroside suppressed the MMP-9 protein expression levels and induced Bax protein expression of bladder cancer cells. (A) Salidroside suppressed MMP-9 and Bax expression, as demonstrated using western blotting; (B) MMP-9 and (C) Bax protein levels were quantified. "P<0.05, compared with the control group. MMP-9, matrix metalloproteinase-9.

in order to induce autophagy (33). In the present study, it was demonstrated that salidroside significantly induced PI3K and
p-Akt protein expression in T24 cells, in a dose-dependent manner, compared with in the control cells $(\mathrm{P}<0.05)$. 
The mRNA expression levels of MMP-2 and MMP-9 are increased in bladder cancer cells transfected with the gene encoding fibroblast growth factor (FGF); however, in cells transfected with the FGF-2 antisense gene, MMP-2 and MMP-9 mRNA levels are decreased, and the invasion force is reduced (34). Thus, MMPs are regarded to be associated with bladder cancer cell infiltration (34). A previous study on the expression levels of MMP-2 and tissue inhibitor of metalloproteinases 2 (TIMP-2) in bladder cancer tissues revealed that MMP-2 and TIMP-2 are highly expressed in cancer tissues; the prognosis of patients with high MMP-2 and TIMP-2 expression levels is significantly poorer compared with that of patients with low expression levels (35). In the present study, salidroside significantly decreased MMP-9 protein expression levels in T24 cells. Sun et al (36) revealed that salidroside inhibited the proliferation of, and decreased the migration and invasion of SW1116 cells through the suppression of MMP-2 and MMP-9 expression. The results of the present study corresponded with those of the prior studies discussed here.

Previous molecular biology studies have demonstrated that the occurrence and development of bladder cancer is associated with cell apoptosis in prostate tissue $(37,38)$. Among the genes involved in the regulation of apoptosis, BCL2 is of particular interest. The product of BCL2 is a $26 \mathrm{kDa}$ protein, localized to the mitochondrial and nuclear membranes, and to the endoplasmic reticulum (37). Previously, it was identified that Bcl-2 may inhibit apoptosis in a variety of tissue types, and it is now widely recognized as an anti-apoptotic gene, participating in tumorigenesis by inhibiting apoptosis and prolonging cell survival (38). A previous study demonstrated that for $69 \%$ of all patients with bladder cancer, BCL2 is positively expressed, and that the expression level is associated with the cancer; in patients with bladder cancer at a progressive stage, the expression of $\mathrm{Bcl}-2$ is $\leq 39 \%$, whereas at a less-progressive stage, it is $\sim 24 \%$ (39). The results of the present study revealed that the administration of salidroside significantly inhibited the Bax signaling pathway and induced caspase-9/3 activation in T24 cells. Lv et al (22) suggested that salidroside reduces renal cell carcinoma proliferation through the suppression of Bax expression.

In summary, the present study demonstrated for the first time, to the best of our knowledge, that salidroside reduces viability and induces apoptosis in T24 human bladder cancer cells through the autophagy/PI3K/Akt and Bcl-2 signaling pathways. Furthermore, the molecular mechanisms underlying the effects of salidroside in human bladder cancer cells were explored. The results suggested that salidroside may be an effective agent for treating human bladder cancer. However, there were a number of limitations on the present study, including using only T24 cells; further in vitro and in vivo models, in addition to clinical studies, will be required to explore the effects in additional cell lines and other cancer types. Future studies should also be conducted using normal cell lines, in addition to other tumor cell lines.

\section{Acknowledgements}

Not applicable.

\section{Funding}

No funding was received.

\section{Availability of data and materials}

The analyzed data sets generated during the study are available from the corresponding author on reasonable request.

\section{Authors' contributions}

TL designed the experiment. KX and YL performed the experiments. TL analyzed the data and wrote the manuscript.

\section{Ethics approval and consent to participate}

Not applicable.

\section{Consent for publication}

Not applicable.

\section{Competing interests}

The authors declare that they have no competing interests.

\section{References}

1. Liu L, Zhao X, Zhu X, Zhong Z, Xu R, Wang Z, Cao J and Hou Y: Decreased expression of miR-430 promotes the development of bladder cancer via the upregulation of CXCR7. Mol Med Rep 8: 140-146, 2013.

2. Palmeira C, Oliveira PA, Lameiras C, Amaro T, Silva VM, Lopes $\mathrm{C}$ and Santos L: Biological similarities between murine chemical-induced and natural human bladder carcinogenesis. Oncol Lett 1: 373-377, 2010.

3. Billerey C, Chopin D, Aubriot-Lorton MH, Ricol D, Gil Diez de Medina S, Van Rhijn B, Bralet MP, Lefrere-Belda MA, Lahaye JB, Abbou CC, et al: Frequent FGFR3 mutations in papillary non-invasive bladder (pTa) tumors. Am J Pathol 158: 1955-1959, 2001

4. Xiao J, Chen JX, Zhu YP, Zhou LY, Shu QA and Chen LW: Reduced expression of MTUS1 mRNA is correlated with poor prognosis in bladder cancer. Oncol Lett 4: 113-118, 2012.

5. Fan Z, Huangfu X and Liu Z: Effect of autophagy on cisplatin caused bladder cancer cell apoptosis. Panminerva Med 59: 1-8, 2016.

6. Pinto-Leite R, Arantes-Rodrigues R, Ferreira R, Palmeira C, Colaço A, Moreira da Silva V, Oliveira P and Lara Santos L: Temsirolimus improves cytotoxic efficacy of cisplatin and gemcitabine against urinary bladder cancer cell lines. Urol Oncol 32: 41 e11-e22, 2014.

7. Lin C, Tsai SC, Tseng MT, Peng SF, Kuo SC, Lin MW, Hsu YM, Lee MR, Amagaya S, Huang WW, et al: AKT serine/threonine protein kinase modulates baicalin-triggered autophagy in human bladder cancer T24 cells. Int J Oncol 42: 993-1000, 2013.

8. Pan XW, Li L, Huang Y, Huang H, Xu DF, Gao Y, Chen L, Ren JZ, Cao JW, Hong Y and Cui XG: Icaritin acts synergistically with epirubicin to suppress bladder cancer growth through inhibition of autophagy. Oncol Rep 35: 334-342, 2016.

9. Du L, Jiang N, Wang G, Chu Y, Lin W, Qian J, Zhang Y, Zheng J and Chen G: Autophagy inhibition sensitizes bladder cancer cells to the photodynamic effects of the novel photosensitizer chlorophyllin e4. J Photochem Photobiol B 133: 1-10, 2014.

10. Gao H, Wang $\mathrm{H}$ and Peng J: Hispidulin induces apoptosis through mitochondrial dysfunction and inhibition of P13k/Akt signalling pathway in HepG2 cancer cells. Cell Biochem Biophys 69: 27-34, 2014.

11. Wallerand H, Ravaud A and Ferrière JM: Bladder cancer in patients after organ transplantation. Curr Opin Urol 20: 432-436, 2010.

12. Lee KR, Lee JS, Kim YR, Song IG and Hong EK: Polysaccharide from Inonotus obliquus inhibits migration and invasion in B16-F10 cells by suppressing MMP-2 and MMP-9 via downregulation of NF-kB signaling pathway. Oncol Rep 31: 2447-2453, 2014. 
13. Zhao M, Hu HG, Huang J, Zou Q, Wang J, Liu MQ, Zhao Y, Li GZ, Xue S and Wu ZS: Expression and correlation of Twist and gelatinases in breast cancer. Exp Ther Med 6: 97-100, 2013.

14. Li Z, Ren Y, Wu QC, Lin SX, Liang YJ and Liang HZ: Macrophage migration inhibitory factor enhances neoplastic cel invasion by inducing the expression of matrix metalloproteinase 9 and interleukin-8 in nasopharyngeal carcinoma cell lines. Chin Med J (Engl) 117: 107-114, 2004.

15. Cappellen D, De Oliveira C, Ricol D, de Medina S, Bourdin J, Sastre-Garau X, Chopin D, Thiery JP and Radvanyi F: Frequent activating mutations of FGFR3 in human bladder and cervix carcinomas. Nat Genet 23: 18-20, 1999.

16. Choi BD, Jeong SJ, Wang G, Park JJ, Lim DS, Kim BH, Cho YI, Kim CS and Jeong MJ: Secretory leukocyte protease inhibitor is associated with MMP-2 and MMP-9 to promote migration and invasion in SNU638 gastric cancer cells. Int J Mol Med 28: 527-534, 2011.

17. Chen ZY, Liang K, Lin Y and Yang F: Study of the UTMD-based delivery system to induce cervical cancer cell apoptosis and inhibit proliferation with shRNA targeting Survivin. Int J Mol Sci 14: 1763-1777, 2013

18. Liu Y, He P, Liu F, Shi L, Zhu H, Cheng X, Zhao J, Wang Y and Zhang M: Prognostic significance of B-cell lymphoma 2 expression in acute leukemia: A systematic review and meta-analysis. Mol Clin Oncol 2: 411-414, 2014.

19. Jiang H, Yu P, Qian DH, Qin ZX, Sun XJ, Yu J and Huang L: Hydrogen-rich medium suppresses the generation of reactive oxygen species, elevates the Bcl-2/Bax ratio and inhibits advanced glycation end product-induced apoptosis. Int $\mathrm{J}$ Mol Med 31: 1381-1387, 2013.

20. Park HY, Kim GY, Moon SK, Kim WJ, Yoo YH and Choi YH: Fucoidan inhibits the proliferation of human urinary bladder cancer T24 cells by blocking cell cycle progression and inducing apoptosis. Molecules 19: 5981-5998, 2014.

21. Fan XJ, Wang Y, Wang L and Zhu M: Salidroside induces apoptosis and autophagy in human colorectal cancer cells through inhibition of PI3K/Akt/mTOR pathway. Oncol Rep 36: 35593567,2016

22. Lv C, Huang Y, Liu ZX, Yu D and Bai ZM: Salidroside reduces renal cell carcinoma proliferation by inhibiting JAK2/STAT3 signaling. Cancer Biomark 17: 41-47, 2016.

23. Li HL, Zhang S, Wang Y, Liang RR, Li J, An P, Wang ZM, Yang J and Li ZF: Baicalein induces apoptosis via a mitochondrial-dependent caspase activation pathway in T24 bladder cancer cells. Mol Med Rep 7: 266-270, 2013.

24. Matsuda T and Hori M: Five-year relative survival rate of bladder cancer in the USA, Europe and Japan. Jpn J Clin Oncol 44: 776, 2014.

25. Mi C, Shi H, Ma J, Han LZ, Lee JJ and Jin X: Celastrol induces the apoptosis of breast cancer cells and inhibits their invasion via downregulation of MMP-9. Oncol Rep 32: 2527-2532, 2014.

26. Fribley AM, Miller JR, Brownell AL, Garshott DM, Zeng Q, Reist TE, Narula N, Cai P, Xi Y, Callaghan MU, et al: Celastrol induces unfolded protein response-dependent cell death in head and neck cancer. Exp Cell Res 330: 412-422, 2015.

27. Buffen K, Oosting M, Quintin J, Ng A, Kleinnijenhuis J, Kumar V, van de Vosse E, Wijmenga C, van Crevel R, Oosterwijk E, et al: Autophagy controls BCG-induced trained immunity and the response to intravesical BCG therapy for bladder cancer. PLoS Pathog 10: e1004485, 2014.
28. Schmitz KJ, Ademi C, Bertram S, Schmid KW and Baba HA Prognostic relevance of autophagy-related markers LC3, p62/sequestosome 1, Beclin-1 and ULK1 in colorectal cancer patients with respect to KRAS mutational status. World J Surg Oncol 14: 189, 2016

29. Lihuan D, Jingcun Z, Ning J, Guozeng W, Yiwei C, Wei L, Jing Q, Yuanfang $Z$ and Gang C: Photodynamic therapy with the novel photosensitizer chlorophyllin $\mathrm{f}$ induces apoptosis and autophagy in human bladder cancer cells. Lasers Surg Med 46: 319-334, 2014.

30. Li H, Sun L, de Carvalho EL, Li X, Lv X, Khan GJ, Semukunzi H, Yuan S and Lin S: DT-13, a saponin monomer of dwarf lilyturf tuber, induces autophagy and potentiates anti-cancer effect of nutrient deprivation. Eur J Pharmacol 781: 164-172, 2016.

31. Zhang C, Jia X, Wang K, Bao J, Li P, Chen M, Wan JB, Su H, Mei Z and He C: Polyphyllin VII Induces an Autophagic cell death by activation of the JNK pathway and inhibition of PI3K/AKT/mTOR pathway in HepG2 cells. PLoS One 11: e0147405, 2016.

32. Lin CJ, Chen TL, Tseng YY, Wu GJ, Hsieh MH, Lin YW and Chen RM: Honokiol induces autophagic cell death in malignant glioma through reactive oxygen species-mediated regulation of the p53/PI3K/Akt/mTOR signaling pathway. Toxicol Appl Pharmacol 304: 59-69, 2016.

33. Cao ZX, Yang YT, Yu S, Li YZ, Wang WW, Huang J, Xie XF, Xiong L, Lei S and Peng C: Pogostone induces autophagy and apoptosis involving PI3K/Akt/mTOR axis in human colorectal carcinoma HCT116 cells. J Ethnopharmacol 202: 20-27, 2017.

34. Fujiwara Y, Shiraya S, Miyake T, Yamakawa S, Aoki M, Makino H, Nishimura $M$ and Morishita R: Inhibition of experimental abdominal aortic aneurysm in a rat model by the angiotensin receptor blocker valsartan. Int J Mol Med 22: 703-708, 2008

35. Eissa S, Shabayek MI, Ismail MF, El-Allawy RM and Hamdy MA: Diagnostic evaluation of apoptosis inhibitory gene and tissue inhibitor matrix metalloproteinase- 2 in patients with bladder cancer. IUBMB Life 62: 394-399, 2010.

36. Sun KX, Xia HW and Xia RL: Anticancer effect of salidroside on colon cancer through inhibiting JAK2/STAT3 signaling pathway. Int J Clin Exp Pathol 8: 615-621, 2015.Perabo FG, Wirger A, Kamp S, Lindner H, Schmidt DH, Müller SC and Kohn EC: Carboxyamido-triazole (CAI), a signal transduction inhibitor induces growth inhibition and apoptosis in bladder cancer cells by modulation of Bcl-2. Anticancer Res 24: 2869-2877, 2004.

37. Yuan SS, Chang HL, Chen HW, Kuo FC, Liaw CC, Su JH and Wu YC: Selective cytotoxicity of squamocin on T24 bladder cancer cells at the S-phase via a Bax-, Bad-, and caspase-3-related pathways. Life Sci 78: 869-874, 2006

38. Zhou C, Ding J and Wu Y: Resveratrol induces apoptosis of bladder cancer cells via miR21 regulation of the $\mathrm{Akt} / \mathrm{Bcl} 2$ signaling pathway. Mol Med Rep 9: 1467-1473, 2014. 